\title{
LA GESTIÓN POR PROCESOS Y SU INCIDENCIA EN LOS COSTOS OPERATIVOS DE LAS INSTITUCIONES PÚBLICAS: SUPERINTENDENCIA NACIONAL DE BIENES ESTATALES
}

\author{
PROCESS MANAGEMENT AND ITS INCIDENCE IN THE OPERATIONAL COSTS OF \\ PUBLIC INSTITUTIONS: NATIONAL SUPERINTENDENCY OF STATE PROPERTY
}

\section{Jussara Inez Anaí Palmer Torres \\ Universidad Nacional Mayor de San Marcos Licenciada en Administración en la Universidad Nacional Mayor de San Marcos Correo electrónico: jpalmert@unmsm.edu.pe}

Juan Victoriano Castillo Maza Universidad Nacional Mayor de San Marcos Doctor en Ciencias Contables y Empresariales en la Universidad Nacional Mayor de San Marcos Correo electrónico: jcastillom@unmsm.edu.pe

[Recibido: 12/07/2018 Aceptado: 08/08/2018]

\section{RESUMEN}

Objetivo: Determinar la incidencia del proceso de Afectación en uso de los predios en la gestión de los bienes inmuebles del Estado administrados por la Superintendencia Nacional de Bienes Estatales, periodo 2012 - 2016. Método: Es una investigación de tipo sustantiva explicativa, cuantitativa, transversal, retrospectiva, correlacional y no experimental, con base en la medición, la evaluación numérica y el análisis estadístico. Resultados: La muestra estuvo constituida por 20 solicitudes, a quienes se les aplicó las pruebas chi-cuadrado y luego se relacionaron las variables; los resultados indicaron que el tiempo de los procedimientos de evaluación, ejecución e inscripción registral sí afecta la gestión de los bienes inmuebles del Estado administrados por la SBN. Conclusiones: La importancia de la Gestión por procesos como piedra angular en el quehacer público es económica y socialmente rentable, contribuyendo así con su modernización. Se mejoró el proceso de Afectación en uso de los predios y se diseñaron el mapa de procesos y la cadena de valor de la SBN, por su impacto sistémico positivo en la gestión de los bienes inmuebles del Estado.

Palabras clave: Gestión por procesos, afectación, uso de predios, mapa de procesos, cadena de valor.

\section{ABSTRACT}

Objective: To determine the incidence of the Affectation process in the use of the properties in the management of the real estate of the State administered by the National Superintendence of State Property, period 2012 - 2016. Method: It is a substantive, explanatory, quantitative, transversal research and a retrospective, correlational and non-experimental one, based on measurement, numerical evaluation and statistical analysis. Results: The sample consisted of 20 applications, to which the chi-square tests were applied and then the variables were related; the results indicated that the time of the evaluation, execution and registration procedures does affect the management of the State's real estate administered by the NATIONAL SUPERINTENDENCY OF STATE PROPERTY (SBN). Conclusions: The importance of Process Management as a cornerstone in public affairs is economically and socially profitable, thus contributing to its modernization. The Affectation process in the use of the properties was improved and the process map and value chain of the SBN were designed, due to its positive systemic impact on the management of the State's real estate.

Keywords: Process management, affectation, land use, process map, value chain. 


\section{INTRODUCCIÓN}

Treflogui (2013) refiere que la gestión pública es el conjunto de acciones conducidas por los aparatos administrativos que buscan efectivizar las políticas públicas, lo cual requiere la utilización de recursos a través de procesos productivos.

En el contexto nacional, diversas instituciones públicas no presentan un desempeño óptimo, y en algunas zonas, su presencia es nula, haciendo notar su incapacidad administrativa. La seguridad y la justicia, funciones básicas del Estado, no son mínimamente garantizadas (Levitsky, 2012).

Estas carencias inciden de manera negativa en el bienestar de los ciudadanos, en la competitividad empresarial, la legitimidad de los gobiernos y, por ende, en el grado de institucionalidad requerido.

El Estado requería la creación de una política integradora de, que permita y asegure la articulación de sus tres niveles de gobierno, en pro de su mejora al servicio de sus ciudadanos. En ese sentido, se aprobó la Política Nacional de Modernización de la Gestión Pública al 2021, de aplicación a las entidades de la Administración Pública como la Superintendencia Nacional de Bienes Estatales (Decreto Supremo No 004-2013 - PCM, 2013).

Su tercer pilar, la Gestión por procesos, es el "enfoque metodológico que permite gestionar integralmente los procesos, actividades, tareas y formas de trabajo contenidas en la cadena de valor, para convertirlas en una secuencia, que asegure que todos los bienes y/o servicios públicos generen resultados e impactos positivos para el ciudadano, en función de los recursos disponibles" (Secretaría de Gestión Pública de la Presidencia del Consejo de Ministros, 2014, p.40).

Se define a la mejora continua de procesos como: "Una metodología sistemática desarrollada para ayudar a una organización a tener avances significativos en la manera que operan sus procesos". (Harrington 1991, p. 20-21 citado en Suárez y Ramis, 2008)

Por su parte, Bravo (2011) describe ciertas características de la mejora de procesos:

> Se busca la perfección del proceso existente, a fin de mejorar su costo, eficiencia, tiempo y calidad de atención.

> La organización es una unidad orientada a la satisfacción del cliente.

> Es importante conocer por qué y para qué se hace de esa manera.

> Se crean equipos de trabajo que realizan y dirigen un proceso orientados al mismo objetivo.

Vega y González (2013) resaltan la trascendencia de la sostenibilidad de la mejora de procesos, como garantía de una adecuada inversión en el cambio, destacando el alto riesgo de las innovaciones en el sector público, dados los vaivenes políticos que la caracterizan. Es así como recomiendan cuatro medidas para garantizar esa sostenibilidad: i) Generar vertiginosas ganancias que sostengan el proceso de mejora, ii) concebir aliados externos, cuya opinión sea aval ante cualquier duda, iii) involucrar a los servidores públicos que implementan la mejora del proceso, y iv) crear candados legales estratégicos y recurrir solo a los estrictamente necesarios.

En el marco de la Ley General del Sistema Nacional de Bienes Estatales - Ley N 29151 y su Reglamento, la SBN es el "ente rector del Sistema Nacional de Bienes Estatales y organismo público ejecutor responsable de normar los actos de adquisición, registro, disposición, administración y supervisión de los bienes estatales; así como de ejecutar dichos actos respecto de los bienes cuya administración está a su cargo, de acuerdo a la normatividad vigente, con representación judicial propia" (Superintendencia Nacional de Bienes Estatales, 2016, p.16).

Esa función rectora, en pro de la supervisión de los bienes estatales y de la administración del Sistema $\mathrm{Na}$ cional de Bienes Estatales, redundará en la aprobación de actos de administración de carácter y alcance nacional, de manera eficaz y eficiente.

Precisamente, la investigación se centrará en uno de los tipos de Actos de Administración de la SBN: La Afectación en uso, que es el derecho de cualquier entidad pública o particular a usar y administrar de manera gratuita un predio de propiedad estatal, por un plazo indeterminado, para un fin establecido y compatible con las funciones del Estado. Tiene como objetivo beneficiar con el uso de propiedad estatal a entidades públicas o privadas que coadyuven al fin social del Estado.

Según consta en el Texto Único de Procedimientos Administrativos - TUPA de la SBN, aprobado por D.S. 
N021-2012-VIVIENDA, el proceso Afectación en uso de los predios tiene un plazo de duración de treinta (30) días, desde la evaluación de la solicitud hasta la suscripción del acta de entrega por afectación en uso y su actualización en el Sistema de Información Nacional de Bienes Estatales.

La realidad en cuanto al tiempo transcurrido difiere completamente de lo contemplado en el Texto Único de Procedimientos Administrativos - TUPA, lo que provoca la insatisfacción de los usuarios (actualmente tarda ciento doce días) y las constantes observaciones que devienen de los Informes de Control del Órgano de Control Interno realizados a la Subdirección de Administración del Patrimonio Estatal - SDAPE, unidad responsable de este proceso.

Cabe mencionar, que la gestión ineficaz e ineficiente de los bienes inmuebles estatales deviene en su pérdida, desvalorización, subutilización y falta de control de parte del órgano rector, traducido en un retroceso en el desarrollo económico del país.

La pregunta de investigación es:

¿De qué manera el proceso afectación en uso de los predios incide en la gestión de los bienes inmuebles del Estado administrados por la Superintendencia Nacional de Bienes Estatales, período 2012 - 2016?

\section{Hipótesis}

El proceso afectación en uso de los predios incide significativamente en la gestión de bienes inmuebles del estado administrados por la Superintendencia Nacional de Bienes Estatales, periodo 2012 - 2016.

\section{Objetivo}

Determinar la incidencia del proceso afectación en uso de los predios en la gestión de los bienes inmuebles del Estado administrados por la Superintendencia Nacional de Bienes Estatales, periodo 2012 - 2016.

\section{MATERIAL Y MÉTODOS}

Hernández, Fernández y Baptista (2010) refieren que el tipo de la investigación está determinado por su nivel y método. En cuanto al nivel, de acuerdo a la naturaleza del problema planteado y objetivos propuestos, la investigación es de tipo sustantiva explicativa y aplicada. Respecto al método empleado, es decir de acuerdo a la estrategia que se utilizó para comprobar las hipótesis formuladas, responde al tipo cuantitativa, transversal y retrospectiva. Asimismo, esta investigación clásica cuantitativa corresponde al tipo analítico, descriptivo, correlacional y no experimental.

El desarrollo de la investigación comprendió la construcción del marco teórico (marco filosófico, antecedentes nacionales e internacionales y bases teóricas) necesario para el adecuado conocimiento e interpretación del proceso Afectación en uso de los predios, a fin de evaluar su incidencia en la gestión de bienes inmuebles del Estado administrados por la Superintendencia Nacional de Bienes Estatales. Seguidamente, se recopiló la información de origen secundario correspondiente al periodo 2012 2016, sobre las variables e indicadores correspondientes. Se realizaron entrevistas y se practicaron dos cuestionarios al usuario interno y externo de la Subdirección de Administración del Patrimonio Estatal de la SBN.

La población de estudio está constituida por el total de solicitudes del servicio de afectación en uso presentadas en Mesa de Partes de la SBN: Año 2012 (11 solicitudes), Año 2013 (9 solicitudes), Año 2014 (74 solicitudes), Año 2015 (106 solicitudes) y Año 2016 (80 solicitudes), haciendo un total de 280 solicitudes.

Tomando como referencia a los mismos autores, se recurrió al "muestreo por conveniencia", y considerando simplemente los casos disponibles a los cuales se tuvo acceso, la muestra será el $7 \%$ de la población de estudio (19,6 solicitudes). Para efectos de la investigación, la muestra será de 20 solicitudes.

\section{RESULTADOS}

Son 248 las actividades que, en suma, conforman el proceso a analizar, desde que el usuario presenta su solicitud en la Unidad de Trámite Documentario (Actividad $\mathrm{N}^{\circ} 0$ ), hasta que el expediente se cierra en el Sistema de Trámite Documentario (Actividad $\mathrm{N}^{\circ}$ 248). Todo ello tarda 53,993 minutos, lo que equivale a 900 horas y ello a su vez a 112 días, distribuidos de la siguiente manera: Evaluación (38 días), Ejecución (58 días) e Inscripción Registral (16 días).

Dado que las hipótesis propuestas toman en cuenta el tiempo de los tres procedimientos que forma parte del proceso, se analizó la documentación recabada, compro- 
bándose que ninguna de las solicitudes que conforman la muestra a investigar, cumple con el tiempo establecido en el TUPA vigente.

Mediante la prueba Chi-Cuadrado, planteando que el tiempo de duración del proceso debería de ser 47 días y la eficiencia igual o superior al $100 \%$, se realizó el análisis de las 20 solicitudes en estudio.
(Ho): El tiempo de los procedimientos Evaluación, Ejecución e Inscripción Registral NO influye en la gestión de los bienes inmuebles del Estado administrados por la SBN.

(Ha): El tiempo de los procedimientos Evaluación, Ejecución e Inscripción Registral afecta la gestión de los bienes inmuebles del Estado administrados por la SBN.

Tabla 1.

Tabla de contingencia tiempo real y eficiencia.

\begin{tabular}{|c|c|c|c|c|}
\hline \multicolumn{5}{|c|}{ Recuento } \\
\hline & & \multicolumn{2}{|c|}{ EFICIENCIA } & \multirow[t]{2}{*}{ Total } \\
\hline & & NO SUPERÓ & SUPERÓ & \\
\hline \multirow{2}{*}{ TIEMPO_REAL } & DENTRO DEL T. MEJORADO & 1 & 9 & 10 \\
\hline & FUERA DEL T. MEJORADO & 10 & 0 & 10 \\
\hline Total & & 11 & 9 & 20 \\
\hline
\end{tabular}

Fuente: Elaboración propia.

Tabla 2.

Pruebas de Chi-Cuadrado.

\begin{tabular}{lcccrr}
\hline & Valor & gl & $\begin{array}{c}\text { Sig. asintótica } \\
\text { (bilateral) }\end{array}$ & $\begin{array}{c}\text { Sig. exacta } \\
\text { (bilateral) }\end{array}$ & $\begin{array}{c}\text { Sig. exacta } \\
\text { (unilateral) }\end{array}$ \\
\hline Chi-cuadrado de Pearson & $16,364^{z}$ & 1 & 000 &, 000 &, 000 \\
\hline Estadístico exacto de Fisher & & & & & \\
\hline N de casos válidos & 20 & & & & \\
\hline
\end{tabular}

Fuente: Elaboración propia.

Como el p-valor $<a(0,000<0,05)$, se rechaza la Ho y se acepta la Ha. Por lo tanto, el tiempo de los procedimientos Evaluación, Ejecución e Inscripción Registral sí afecta la gestión de los bienes inmuebles del Estado administrados por la SBN.

Nótese, que los resultados arrojados por el SPSS muestran que el $50 \%$ de casillas tienen una frecuencia espera- da inferior a 5, cuando lo máximo permitido es $20 \%$. En este caso, se recurrió al Test-exacto de Fisher, dado que se están analizando variables dicotómicas. Dado que su valor es 0 , menor a la significancia que es 0,05 ; la prueba es válida. 


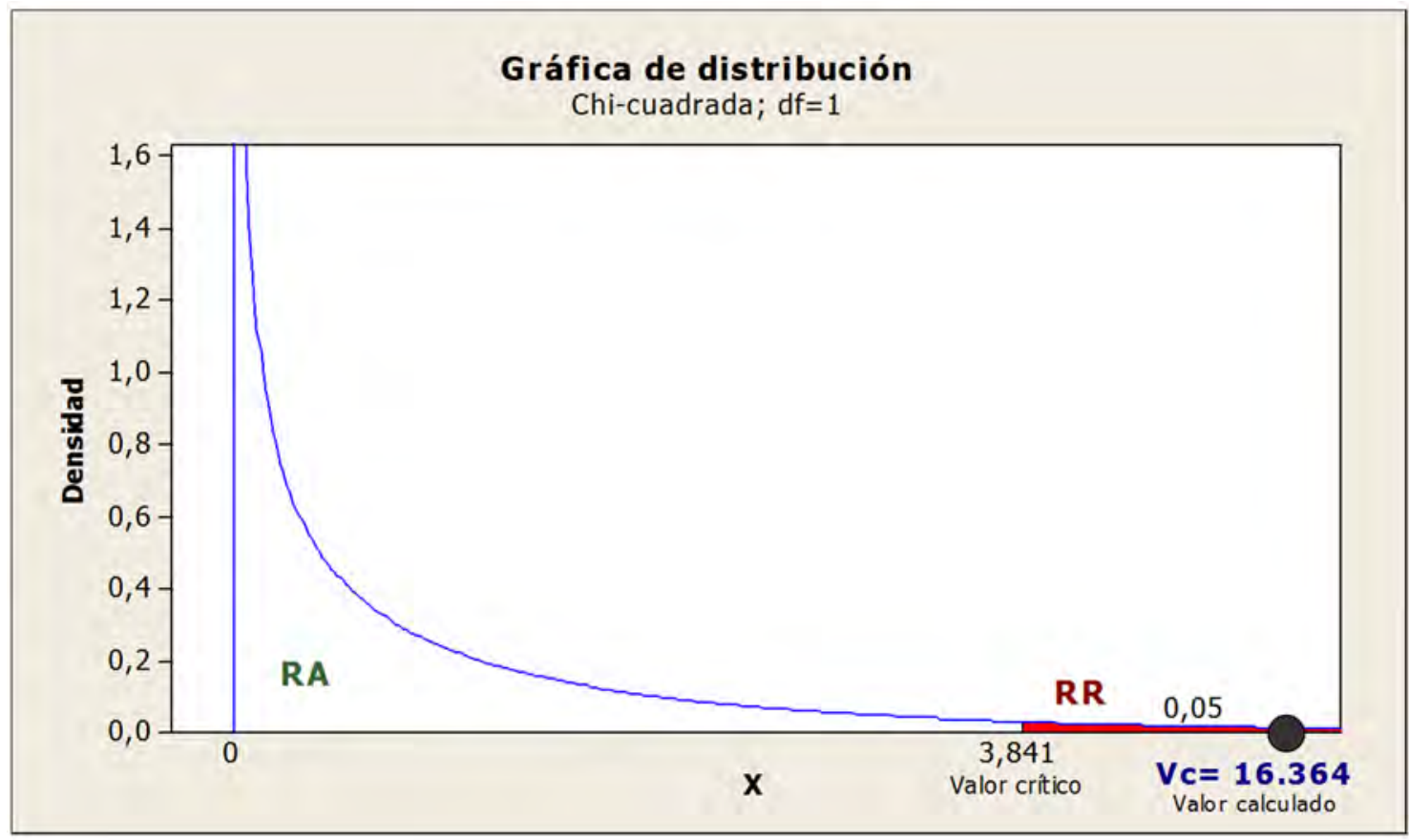

Figura 1. Gráfica de distribución. Chi-Cuadrado. Fuente: Elaboración propia.

También, se analizaron las dimensiones Eficacia y Eficiencia, al ser los indicadores de la variable dependiente. Se determinó cuán eficaz ha sido la SDAPE en el periodo 2012 - 2016, respecto al cumplimiento del número proyectado de predios afectados en uso para cada año fiscal. Considerando que la eficacia óptima debe ser el 100\%, se encontró que en el año 2012 la SDAPE logró ser más eficaz (eficacia alcanzada $=175 \%$ ), puesto que proyectó afectar en uso 16 predios, y logró afectar en uso un total de 28 predios.

Del otro lado, el año en que la SDAPE logró el menor porcentaje de eficacia fue en el 2015 (eficacia alcanzada = $42 \%$ ), puesto que proyectó afectar en uso 45 predios, pero solo logró afectar un total de 19 predios.

Por su parte, la eficiencia fue calculada por cada caso, tomando como referencia el monto ejecutado en un caso ideal para Lima y otro para provincia.

A fin de hallar el costo diario que la SDAPE destina a un proceso en Lima, se tomó como línea base el caso de menor duración, tal es el caso del Despacho Presidencial. Ello hizo un valor diario promedio de S/ 36,04 soles (S/ 1 297,44 / 36 días). El mismo procedimiento se siguió para un caso en provincia, tomando como referencia el predio solicitado por el MVCS. Ello hizo un valor diario promedio de S/ 175,78 soles (S/ 3 867,16 / 22 días).

Del mismo modo, se tomó en cuenta el tiempo del proceso mejorado, que representa la propuesta de la investigación: 47 días. Considerando que la eficiencia óptima debe ser el $100 \%$, se puede afirmar que el caso menos eficiente fue el del predio solicitado por el INPE (duración de 761 días), cuya eficiencia fue del $6,18 \%$, requiriendo así una mayor asignación de recursos ( $\mathrm{S} / 27$ 426,44 soles).

En contraste, el caso más eficiente fue el del predio solicitado por el MCVS (duración de 22 días), cuya eficiencia fue del 213,64\%, requiriendo la menor asignación de recursos (S/ 3867,16 soles).

Asimismo, se aplicaron dos cuestionarios, recurriendo en ambos casos al uso de la Escala de Likert, con cinco posibles respuestas: Muy satisfecho 5, Satisfecho 4, No sabría que decir 3, Poco satisfecho 2, Insatisfecho 1. El primer cuestionario fue realizado al personal de la SDAPE, estos son el Asesor Técnico, Asesor Legal, Asistente y siete profesionales de línea (Ver Tabla 3). 
Tabla 3.

Resultados de la encuesta que revela el grado de satisfacción laboral de los trabajadores de la SDAPE.

\begin{tabular}{|c|c|c|c|c|c|}
\hline Preguntas & $\begin{array}{c}\text { Muy } \\
\text { satisfecho }\end{array}$ & Satisfecho & $\begin{array}{l}\text { No } \\
\text { sabría } \\
\text { decir }\end{array}$ & $\begin{array}{l}\text { Poco } \\
\text { satisfecho }\end{array}$ & Insatisfecho \\
\hline $\begin{array}{l}\text { ¿Respecto al servicio de Afectación } \\
\text { en uso de predios, se encuentra } \\
\text { conforme con su trabajo y funciones } \\
\text { asignadas? }\end{array}$ & $0 \%$ & $30 \%$ & $0 \%$ & $30 \%$ & $40 \%$ \\
\hline $\begin{array}{l}\text { ¿Se encuentra a gusto con la } \\
\text { infraestructura de la SBN? }\end{array}$ & $0 \%$ & $0 \%$ & $0 \%$ & $20 \%$ & $80 \%$ \\
\hline $\begin{array}{l}\text { ¿Está conforme con el equipamiento } \\
\text { y recursos informáticos proveídos por } \\
\text { la SBN para el desempeño de sus } \\
\text { funciones? }\end{array}$ & $0 \%$ & $0 \%$ & $0 \%$ & $30 \%$ & $70 \%$ \\
\hline $\begin{array}{l}\text { ¿Cree usted que su remuneración } \\
\text { económica es acorde con las } \\
\text { funciones que desempeña? }\end{array}$ & $0 \%$ & $20 \%$ & $0 \%$ & $10 \%$ & $70 \%$ \\
\hline $\begin{array}{l}\text { ¿Le parece adecuada la carga horaria } \\
\text { y laboral asignada? }\end{array}$ & $0 \%$ & $0 \%$ & $20 \%$ & $10 \%$ & $70 \%$ \\
\hline $\begin{array}{l}\text { ¿La dirección y comunicación con sus } \\
\text { superiores es adecuada? }\end{array}$ & $30 \%$ & $0 \%$ & $0 \%$ & $70 \%$ & $0 \%$ \\
\hline $\begin{array}{l}\text { ¿La solución de problemas, por parte } \\
\text { de sus superiores, recurre a la } \\
\text { innovación y mejora continua? }\end{array}$ & $20 \%$ & $10 \%$ & $0 \%$ & $70 \%$ & $0 \%$ \\
\hline $\begin{array}{l}\text { ¿La comunicación con sus superiores } \\
\text { y entre sus compañeros es fluida? }\end{array}$ & $10 \%$ & $20 \%$ & $20 \%$ & $20 \%$ & $30 \%$ \\
\hline $\begin{array}{l}\text { ¿Cree usted que sus sugerencias son } \\
\text { tomadas en cuenta en la toma de } \\
\text { decisiones? }\end{array}$ & $10 \%$ & $20 \%$ & $0 \%$ & $30 \%$ & $40 \%$ \\
\hline $\begin{array}{l}\text { ¿Tiene conocimiento acerca de los } \\
\text { pasos y tiempos establecidos por } \\
\text { cada actividad, a fin de que realice } \\
\text { sus actividades de manera eficaz y } \\
\text { eficientemente? }\end{array}$ & $0 \%$ & $0 \%$ & $30 \%$ & $0 \%$ & $70 \%$ \\
\hline $\begin{array}{l}\text { ¿Fue capacitado en el último año, a } \\
\text { modo de fortalecer sus capacidades? }\end{array}$ & $20 \%$ & $10 \%$ & $20 \%$ & $30 \%$ & $20 \%$ \\
\hline $\begin{array}{l}\text { ¿Se le proporciona información } \\
\text { oportuna y adecuada de su } \\
\text { desempeño y resultados alcanzados } \\
\text { para la mejora continua del servicio? }\end{array}$ & $0 \%$ & $30 \%$ & $0 \%$ & $0 \%$ & $70 \%$ \\
\hline $\begin{array}{l}\text { ¿En general, se encuentra conforme } \\
\text { con el apoyo brindado por la SBN } \\
\text { para el desarrollo de sus funciones? }\end{array}$ & $0 \%$ & $20 \%$ & $10 \%$ & $0 \%$ & $70 \%$ \\
\hline $\begin{array}{l}\text { ¿En general, está usted conforme con } \\
\text { el apoyo brindado por la SDAPE? }\end{array}$ & $20 \%$ & $10 \%$ & $40 \%$ & $30 \%$ & $0 \%$ \\
\hline
\end{tabular}

Fuente: Elaboración propia. 
Los resultados revelan graves problemas internos relacionados con el clima laboral, puesto que del íntegro de encuestados $(10$ personas $=100 \%)$, el $70 \%$ se encuentra poco satisfecho e insatisfecho.

El segundo cuestionario fue practicado a las institu- ciones públicas que solicitaron el servicio, durante el periodo 2012- 2016: Despacho Presidencial, CGBVP, AATE, INGEMMET, MINAM, MVCS, INPE, Municipalidad Distrital de La Molina, MINEDU y el Ministerio Público - Fiscalía de la Nación. (Ver Tabla 4).

Tabla 4

Resultados de la encuesta que revela el grado de satisfacción del usuario externo de la SDAPE.

\begin{tabular}{|c|c|c|c|c|c|}
\hline Preguntas & $\begin{array}{c}\text { Muy } \\
\text { satisfecho }\end{array}$ & Satisfecho & $\begin{array}{l}\text { No } \\
\text { sabría } \\
\text { decir }\end{array}$ & $\begin{array}{c}\text { Poco } \\
\text { satisfecho }\end{array}$ & Insatisfecho \\
\hline $\begin{array}{l}\text { ¿Está satisfecho con el trato recibido en } \\
\text { la Superintendencia Nacional de Bienes } \\
\text { Nacionales? }\end{array}$ & $30 \%$ & $10 \%$ & $0 \%$ & $20 \%$ & $40 \%$ \\
\hline $\begin{array}{l}\text { ¿Se sintió a gusto con la confianza } \\
\text { brindada por el personal de la SDAPE al } \\
\text { momento de presentar su solicitud? }\end{array}$ & $0 \%$ & $30 \%$ & $10 \%$ & $40 \%$ & $20 \%$ \\
\hline $\begin{array}{l}\text { ¿Está conforme con la forma de } \\
\text { administrar el servicio de Afectación en } \\
\text { uso de los predios? }\end{array}$ & $10 \%$ & $10 \%$ & $30 \%$ & $30 \%$ & $20 \%$ \\
\hline $\begin{array}{l}\text { ¿Se encuentra conforme con la calidad } \\
\text { de la atención por parte de la SDAPE en } \\
\text { cuanto oportunidad, tiempo y } \\
\text { efectividad? }\end{array}$ & $20 \%$ & $20 \%$ & $10 \%$ & $20 \%$ & $30 \%$ \\
\hline $\begin{array}{l}\text { ¿Está conforme con el tiempo de espera } \\
\text { para la prestación del servicio? }\end{array}$ & $40 \%$ & $0 \%$ & $0 \%$ & $0 \%$ & $60 \%$ \\
\hline $\begin{array}{l}\text { En términos generales, ¿Su institución } \\
\text { se sintió satisfecha con la atención } \\
\text { recibida? }\end{array}$ & $30 \%$ & $10 \%$ & $0 \%$ & $20 \%$ & $40 \%$ \\
\hline
\end{tabular}

Fuente: Elaboración propia.

Como se puede apreciar en la Tabla 4, del total de instituciones públicas consultadas $(10=100 \%)$, el $60 \%$ se encuentra poco satisfecha e insatisfecha con la prestación del servicio y se debe básicamente al tiempo de espera, que sobrepasa lo estipulado en el TUPA, es decir los 30 días.

El excesivo tiempo incide negativamente en el grado de satisfacción del usuario externo, afectando de manera directa la imagen de la SBN como ente rector del Sistema Nacional de Bienes Estatales. Se puede afirmar que la gestión de la SBN, a través de la SDAPE, no es eficaz y eficiente, puesto que no logra el objetivo en el tiempo establecido (30 días) y destina una mayor cantidad de recursos a la consecución del objetivo (horas hombre y servicios básicos) .
Como aporte, se elaboró el Mapa de Procesos de la SBN (Ver Figura 1), donde uno de los cuatro macroprocesos se denomina "Gestión de bienes estatales", el cual comprende tres subprocesos y ocho procedimientos, enmarcados a su vez en tres procesos transversales: "Gestión de relación con el administrado", "Gestión y operación del servicio" y "Gestión y operación de respuesta”.

Adicionalmente, se diseñó la Cadena de Valor (Ver Figura 2), y sugiere como proceso central la Gestión del registro. Las actividades primarias están representadas por la "Gestión de bienes estatales", "Gestión normativa de bienes estatales", "Gestión para el desarrollo de competencias técnicas de bienes estatales" y "Gestión de supervisión de bienes estatales". Las actividades secundarias comprenden la "Adquisición y saneamiento", "Administración" y "Disposición". 


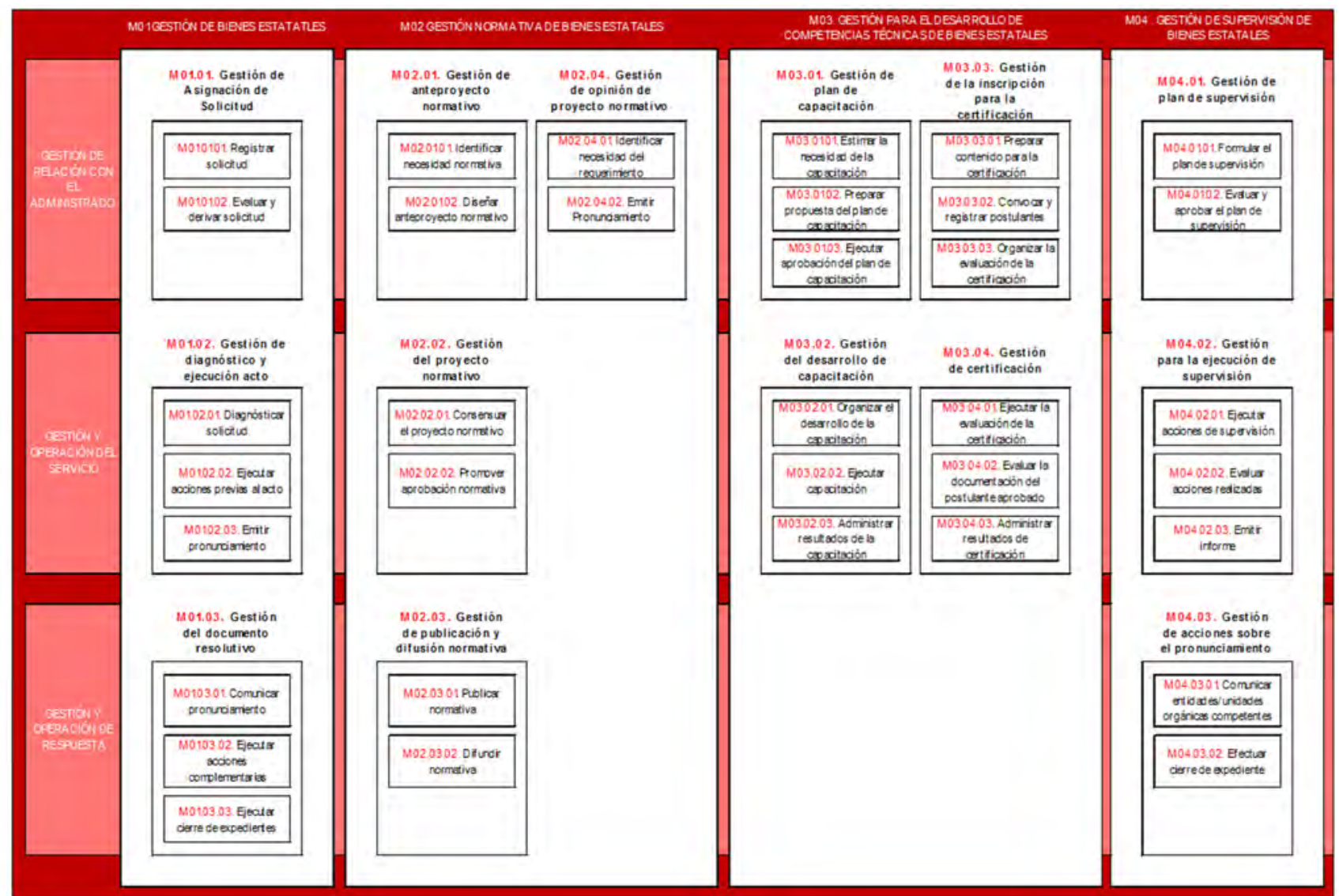

Figura 2. Propuesta de Mapa de Procesos de la SBN.

Fuente: Elaboración propia.

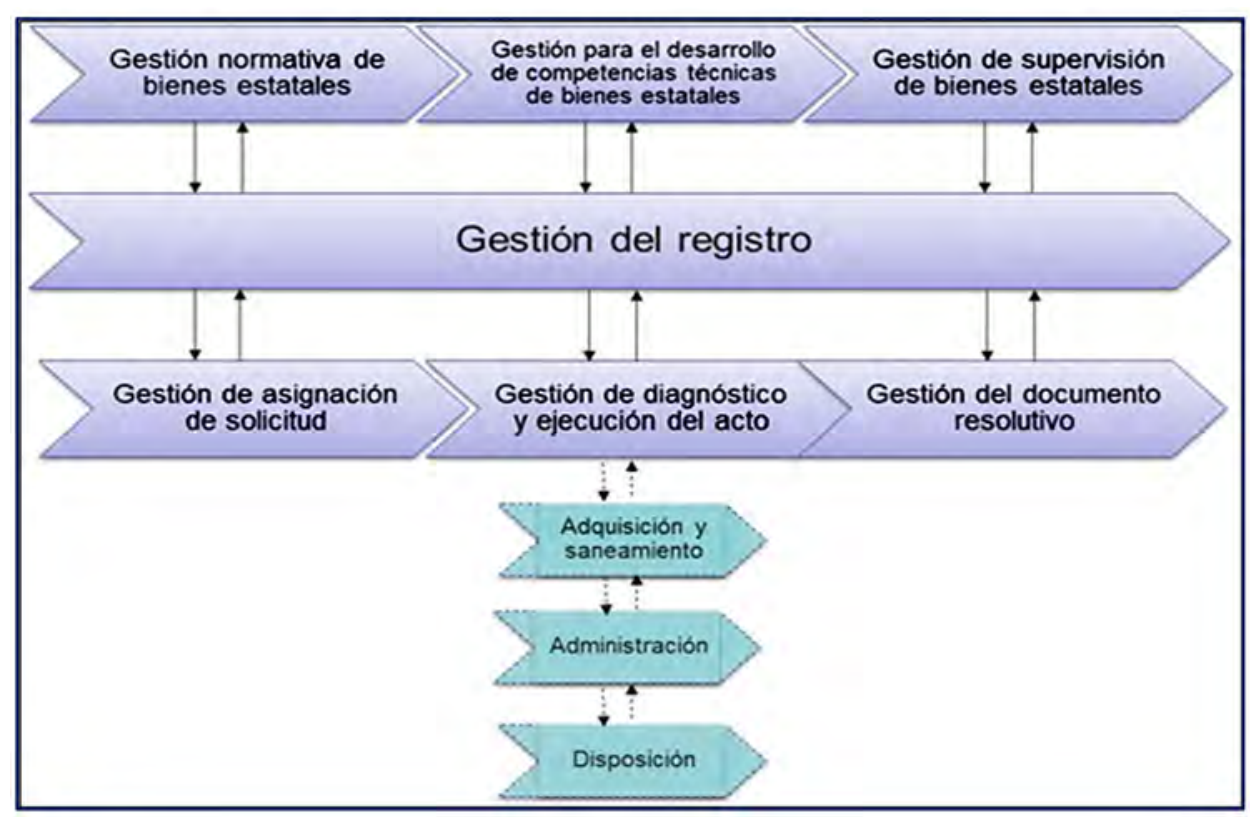

Figura 3. Propuesta de Cadena de Valor de la SBN.

Fuente: Elaboración propia. 
Finalmente, se mejoró el proceso, disminuyéndose los tiempos de espera, fusionando y eliminando actividades que no aportaban valor; concluyéndose que el proceso debe durar 47 días (Ver Figura 3): Evaluación: 103 actividades (16 días), Ejecución: 42 actividades (27 días) e Inscripción Registral: 22 actividades (4 días).

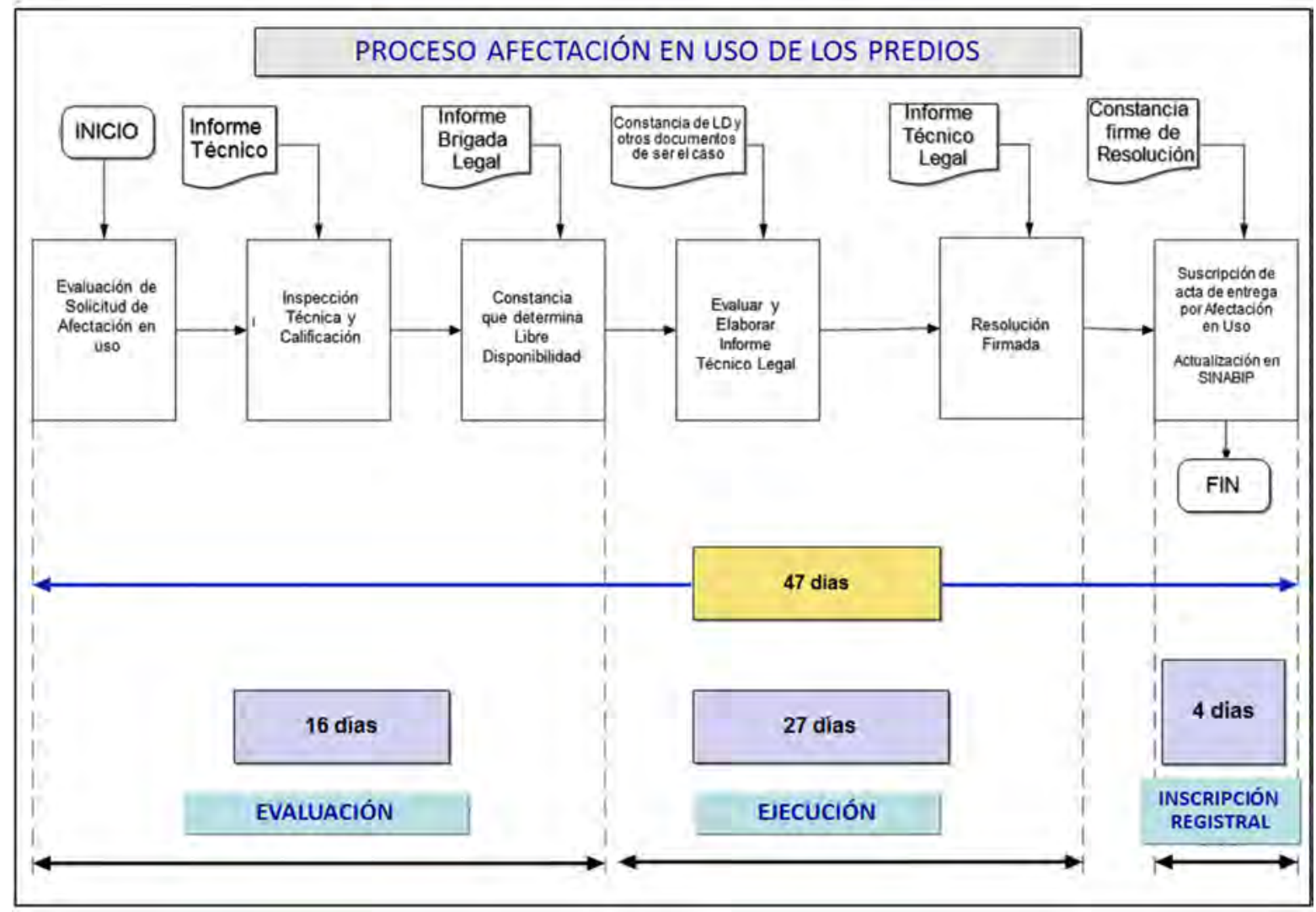

Figura 4. Diagrama de Flujo de Afectación en uso de los predios.

Fuente: Elaboración propia.

\section{DISCUSIÓN}

Se evidenció un número excesivo de actividades que no generan valor agregado al proceso Afectación en uso de los predios, redundando en largos periodos de respuesta al usuario externo, que están fuera de los marcos de referencia y generan el uso ineficiente de los recursos presupuestales asignados. En concordancia con Rengifo (2014), existe una marcada insatisfacción por parte de los usuarios con el servicio brindado por la SBN, que requiere de su pronta y efectiva intervención.

La implementación de la gestión por procesos debe ser progresiva, iniciando con los procesos más importantes y/o los que contribuyan en mayor medida a la misión de la entidad (Carranza, 2016). Es así como la mejora del proceso permitió tener una visión horizontal de la SBN, otorgando una cadena de valor público y valor agregado al servicio ofrecido.
La mejora de la gestión pública, a través de la mejora continua de procesos, sustentada en la eficacia y eficiencia, ha sido adquirida por países como España, con el propósito de enfrentar los factores de cambio, permitiendo así recuperar su legitimidad frente a los ciudadanos (Suárez, 2007). De acuerdo con el autor, se requiere un Estado constituido por instituciones alertas y activas que, además de salvaguardar lo logrado en materia de gestión por procesos, deben innovar continua y sostenidamente; cumpliendo así con los requerimientos de sus stakeholders. Solo así continuará rigiendo su función primigenia: Agregar valor público a la sociedad.

Según lo señalado, se puede concluir que el proceso Afectación en uso de los predios incide significativamente en la gestión de bienes inmuebles del estado administrados por la Superintendencia Nacional de Bienes Estatales, periodo 2012 - 2016. 


\section{REFERENCIAS BIBLIOGRÁFICAS}

Bravo, J. C. (2011). Gestión de Procesos (Alineados con la estrategia). Santiago de Chile: Editorial Evolución S.A.

Carranza, V., Valverde, V., \& Vera, I. (2016). Implementación de la Gestión por Procesos en la Escuela Militar de Chorrillos Coronel Francisco Bolognesi - EMCH CFB (Tesis de Maestría). Universidad del Pacífico, Lima.

Hernández, R., Fernández, C., \& Baptista, P. (2010). Metodología de la Investigación. México: Mc Graw Hill.

Levitsky, S. (2012/05/13). Una paradoja peruana. La República, p. 7 .

Superintendencia Nacional de Bienes Estatales (2016). Compendio Normativo sobre Bienes Inmuebles del Sistema Nacional de Bienes Estatales. Lima: Solvima Graf S.A.C.

Decreto Supremo No 004-2013 - PCM (2013). Política Nacional de Modernización de la Gestión Pública (publicado el 2013/Enero/09). Diario Oficial El Peruano.

Secretaría de Gestión Pública de la Presidencia del Consejo de Ministros (2014). Documento Orientador: Metodología para la Implementación de la Gestión por Procesos en las Entidades de la Administración Pública en el marco del D.S. Nº04-2013-PCM - Política Nacional de Modernización de la Gestión Pública. Obtenido de http://sgp.pcm.gob.pe/wp-content/uploads/2015/03/ Metodologia_de_GxP.pdf

Rengifo, M., Velásquez, C., \& Zavala, U. (2014). Gestión por Procesos: Análisis y mejora del proceso logístico de adquisición de bienes y servicios por una institución del Estado. (Tesis de Maestría). Universidad ESAN, Lima.

Suárez, B. M. (2007). La Sostenibilidad de la Mejora Continua de Procesos en la Administración Pública: Un estudio en los Ayuntamientos de España (Tesis de Doctorado). Universidad Ramón Lull, Barcelona.

S

uárez, B. M., \& Ramis, P. J. (2008). Aplicación y Evolución de la Mejora Continua de Procesos en la Administración Pública. Revista de Globalización, Competitividad y Gobernabilidad, 2(1), 74-83.

Treflogui, G. W. (2013). Gestión Estratégica y Creación de Valor en el Sector Público: Estudio de caso sobre el desarrollo de evaluaciones de conocimientos en el Servicio Civil Peruano (2008 - 2012). (Tesis para optar Título Profesional de Licenciado en Gestión Pública). Pontificia Universidad Católica del Perú, Lima.

Vega, S. C., \& González, E. C. (2013). Modernización y Gerencia Pública. Revista de Administración de Empresas FGV - EAESP, 53(5), 512-518. 